\title{
How networked organisations build capacity for anticipatory governance in South East Asian deltas
}

\author{
Lucas Somavilla Croxatto*, Daniel Hogendoorn, Arthur C. Petersen \\ Department of Science, Technology, Engineering and Public Policy (STEaPP), University College London (UCL), United Kingdom
}

\section{A R T I C L E I N F O}

\section{Keywords:}

Global networks

Anticipatory governance

Capacity building

Project-based interactions

Modelling technologies

Environmental planning

\begin{abstract}
A B S T R A C T
Building capacity for governments to make inferences about future developments enhances their ability to anticipate and plan for climate change adaptation. This study examines the question: how do networked organisations build capacity for anticipatory governance through project-based interactions? We analyse a global network of organisations that mobilise climate and hydrological modelling technologies into the Chao Phraya and Ayeyarwaddy deltas. The methodology innovatively combines ethnographic data with policy analysis and social network analysis. Findings suggest that organisations consolidate technology and knowledge transfer through a global network. However, their governance effect in enhancing anticipatory decision making is found to be marginal at the local level. We argue that anticipatory governance practices need a balancing of foresight tools and techniques with local institutional arrangements in order to be effective. We further demonstrate that technology transfer projects need to be backed up with social and strategic capacity building in order to nurture consistent anticipatory governance in different cultural contexts. We conclude that preventive actions, together with transparent operational response frameworks, could significantly improve resilience and adaptability of local knowledge systems and institutions dealing with climate change adaptation. Such integration could enable anticipatory response measures to better manage risk, as well as increase institutional cooperation for long term environmental planning.
\end{abstract}

\section{Introduction}

Global networks are increasingly associated with the rapid mobilisation of expert knowledge in environmental governance. However, future studies have given little attention to date to how global expertise and their tools (e.g. technologies and policies) influence environmental planning in culturally different places of the world. Unresolved questions about the effectiveness of the transfer of western knowledge and technologies call for further research into increasingly critical areas of inquiry, such as climate change adaptation (Mahoney \& Hulme, 2012). In this study, we address the question: how do networked organisations build capacity for anticipatory governance through project-based interactions? We associate the concept of expertise with foresight (Sardar, 2010), and with what is referred to as anticipatory governance (Fuerth \& Faber, 2012), that is, the capacity of particular actors to envision, imagine, evaluate and strategically respond to anticipated events (Boston, 2017). The study acknowledges that anticipation practices occur under post-normal science conditions and involve complex systems (Funtowicz \& Ravetz, 1994; Young, 2017).

Climate change will have a severe and inevitable impact upon the flood risk of coastal cities (Aerts, Botzen, Bowman, Ward, \&

\footnotetext{
* Corresponding author at: Department of Science, Technology, Engineering and Public Policy (STEaPP), UCL, Euston House (8th Floor), 24 Eversholt Street, London, NW1 1BS, United Kingdom.

E-mail address: lucas.somavilla@ucl.ac.uk (L.S. Croxatto).
} 
Dircke, 2012). In particular, geographical delta regions are dynamic and densely populated environments characterised by intense agricultural activities, rapid development and high vulnerability to climate change. In our research, we contextualised global networks deploying climate and hydrological modelling tools and techniques into deltas located in South East Asia. We argue that anticipatory capacity allows organisations to make more elaborate and robust inferences on matters playing out at different temporal and spatial scales. The advancement of such capacities can be observed in the case of institutions dedicated to water governance on how they plan for a variety of climatic and economic futures in urbanised deltas in South East Asia (Hogendoorn et al., 2018).

At both large and small scales, governments increasingly invest in making representations of possible scenarios that allow better inferences to plan for adaptation to climate change (Enserink, Kwakkel, \& Veenman, 2013; Hajer \& Pelzer, 2018; Kypreos \& Turton, 2011; Vervoort \& Gupta, 2018). In climate adaptation research, it is commonly agreed that human systems adaptation is the process of adjustment to actual or expected climate and its effects, in order to moderate harm or exploit beneficial opportunities (IPCC, 2018). In natural systems, the process of adjustment to actual climate change and its effects can be facilitated, to a certain extent, by human interventions. In this context, we consider the concept of 'adaptive capacity' to be relevant. This concept refers to the ability of systems, institutions and humans, as well as other organisms, to adjust to potential damage, to take advantage of opportunities and to respond to consequences (IPCC, 2014). In complex systems, there are significant challenges with respect to governing adaptation because of the high levels of connectivity, non-linearity and interdependence, which affect the directions of change (Young, 2017).

Thailand and Myanmar offer particularly relevant cases for analysis. Thailand has received international attention in recent years due to the 2011 disastrous floods that affected the city of Bangkok. These floods have been considered as the worst in 50 years (World Bank, 2012). With climate change increasing the likelihood of flooding and storm surge in the deltas of the world, the international cooperation sector has put the focus on finding ways of reducing flood risks in deltas. Myanmar faces conditions similar to Thailand. However, the historical and political situation of the country is very different. Having had a colonial past and a period of isolation due to a military regime, the country has recently opened up its borders (since 2011). The cyclone 'Nargis' in 2008, which had disastrous consequences for Myanmar, was a wake up call for the development cooperation sector to further engage in activities aimed at reducing the country's vulnerability to the effects of climate change. The government has been making significant efforts to address disaster and climate change risk by finalising the Action Plan for Disaster Risk Reduction.

Both countries diverge in term of modes of water control, histories of external intervention and governance arrangements, for example the extent of political decentralisation, high institutional fragmentation, regulatory regimes, urbanisation and industrialisation trends. In this article, we do not attempt to cover all these issues. Instead, we focus on the governance landscape around water and climate change adaptation and analyse the dynamics of networked organisations through project-based interactions. In our research, the notion of project-based interactions is a proxy to address time and place of relational links between networked organisations implementing projects (e.g. between 2016-2019). In an exploratory way, we track and evaluate how operational strategies and technology transfer projects are done in practice.

\subsection{Networked organisations and anticipatory governance}

Collaborative networked capability is argued to be a significant contributor to organisations' competitive advantage (Arkadani, Hashemi, \& Razazzi, 2019). We use the concept of 'networked organisations' to refer to temporal conditions that happen when: "a variety of autonomous organisations, geographically distributed and heterogeneous in terms of their operative environment, culture, social capital and business goals collaborate to achieve common or compatible goals better, and these interactions are supported by computer networks" (Camarinha-Matos \& Afsarmanesh, 2005). Thus, networked organisations - in the plural - form an interconnected system that is formally interrelated for a period of time and with a common set of objectives. We refer to such a system as being a 'cluster'. This article elaborates that climate change adaptation practices involve networked organisations that attempt to provide the capacity to anticipate expected climate and its effects. We argue that networked organisations that participate in technology transfer activities tend to be exclusive - taking part in a specific project and technology-related policy process, which is required to receive official recognition and be considered to have legitimised expertise by other network members.

There is much to learn about how organisations build capacity over time and make use of networks in a global climate governance context which is highly fragmented (van Asselt, 2013). Three key factors have increased communication mechanisms over the past decades: the development of ICT, advances in transport infrastructure and the emergence of global networks, in their various forms, including policy networks (Betsill, Bulkeley, \& Mcguirk, 2004; Haas, 1992; Stone \& Maxwell, 2005), scientific networks, alliances and networks of experts (Gupta, Biermann, Andresen, \& Bernd, 2012), and public and private arrangements (Ottaway, 2001; Wadell, 2011). This phenomenon has been conceptualised in the literature as 'global knowledge networks' (Stone, 2003, 2008). Global knowledge networks account for the increasingly emergent, interconnected and yet ungoverned spaces between the sites of knowledge production and the sites of knowledge application. In our study, we specifically look at a hybrid network that incorporates a range of expert domains, including scientific knowledge, technology, engineering, finance and environmental planning. We emphasise that global networks that build inferential capacity are increasingly diverse and with changing functioning dynamics, where strategy and expertise have become the dominant drivers. These dynamics are not straightforward and require further elucidation.

Consequently, the approach is to put attention on the tools (e.g., models) and techniques (e.g., institutional operations, interpreting data, knowledge management and scenario planning) that together constitute elements of expertise in anticipatory governance (Guston, 2014). For example, modelling technologies combined with expert knowledge support forecast of immediate variability in climate and hydrological systems, but also attempt to generate foresight for informed environmental planning (Vermeulen et al., 2013). Thus, the use of technologies, combined with expertise, can generate performative actions of authoritative knowledge, as they serve the practice of steering possible futures and imagining the potential for change, evaluating assumptions and 
experimenting with new approaches (Vervoort \& Gupta, 2018). The rapid expansion of connectivity affects the development and mobilisations of networked organisations (Scheel, 2002). This development results in active networks having central organisations (as an indicator of strategic behaviour and influence) and others which remain more marginal. We argue that a closer look at both the global patterns of network structures and the relational settling of ties between organisations through the use of tools and techniques allows distinguish between different strategies used by organisations to gain influence and build sound knowledge in anticipatory governance.

\subsection{Capacity building through formal and relational mechanisms}

This study approaches the concept of capacity as both a formal and a relational property embedded in networked organisations. The mobilisation of expertise across organisations involves overcoming the knowledge boundaries between groups, which enables capacity to develop (Clark et al., 2016; van Kerkhoff, 2013). Such mobilisation requires patterns of interactions that include actors, technologies and institutional settings. A concept that grasps the process involving various types of actors that are not formally part of a governmental arrangement is that of 'de facto governance' (Gupta \& Möller, 2019). It includes that actions affect collective outcomes. Hence, in some contexts where sources of governance may not have the intention to govern an event directly, they still generate a governing effect (Rip, 2010). The concept opens up methodological opportunities to triangulate and complement methods that bring actions and interactions into focus (e.g., multi-sited ethnography) with analytical methods that examine patterns that evidence the existence of preceding actions and interaction (e.g., social network and policy analysis). This approach allows distinguishing between formal and relational mechanisms. Formal mechanisms of governance are defined as organisational and legal features such as corporate ownership, structural design, and legally binding contracts (e.g., strategic alliances and joint ventures). Relational mechanisms, on the other hand, are forms of governance which rely upon the social ties created by actions and trust from prior experiences between partners, constituting de facto governance practices. We use the concept of 'de facto governance' as a heuristic that guides our analyses of the informal elements embedded in the activities of networked organisations. Relational mechanisms highlight the importance of interpersonal relations and the development of trust across institutions, acting as an essential building block of collaborative platforms (Fischer, 2015). Formal and relational mechanisms can operate in a complementary fashion. As the study shows, social networks underpinning strategic behaviour can de facto influence the creation of new ties, but also affect the design, governance and consolidation of partnerships.

Relational mechanisms enable repeated interactions and increase spaces in which to govern the development of inferential capacity over time. Most organisations dealing with the implementation of climate and hydrological expertise in South East Asia encounter significant challenges to generate linkages to policy and planning efforts. However, a particular set of networked organisations with a high degree centrality, labelled the 'Hydroclimate cluster' by us, has played a strategic role in building capacity for anticipatory governance through project-based interactions. The analysis shows that particular alliances arise between networked organisations around trusted, authoritative actors that collaborate to build a legitimate transfer of inferential capacity across organisations. Such alliances over time allow for the actors to target strategic directions designed to maintain and sustain frequent interactions, generating governance effects on the way in which expert knowledge is built and gains authority. A relational property of trust over time enhances feasibility prospects and opens up situations for ungoverned spaces to be governed, not by shared norms, institutional arrangements and formal rules, but through strategic engagement and the continuous performance of expertise that links inferential capacity with governance arrangements in generating anticipation.

\section{Methodology}

\subsection{Data collection}

We collected data through fieldwork interviews and desktop research, developing a multi-sited ethnography and an innovative strategy to map global connections (Fischer, 2015; Marcus, 1995; Morita, 2014; Tsing, 2005). We selected respondents through a snowball sampling method and by approaching representatives of organisations found on the internet and in government documents. The web-based search prioritised active project-based operations, considering only organisations linked to expertise in climate and hydrological modelling technologies. We used the following keywords to map the activities of organisations: [climate change, climate modelling, adaptation, hydrology, hydrological modelling, capacity building, development and aid, technology transfer, policy and planning, South East Asia, and implementation]. We conducted 82 interviews with professionals between April 2016-January 2019. Our fieldwork took place in multiple locations in Thailand, Myanmar, The Netherlands, the United Kingdom, and Denmark. Further Skype interviews took place with respondents in Australia, Singapore and Japan. Interviews were practice-focused and semi-structured, with questions aiming to grasp a) the connections between organisations, b) the strategies and aims of organisations and their operations, and c) specific significant actions and events, remembered as conveyed by vignettes. The interviews were recorded in audio form and complemented with field notes. Secondary data included official reports, policies and plans, as well as implementation projects, which were compared by examining formal connections between organisations and making a network representation for analysis. We focused on formal connections, that is, interactions that have legal documentation, such as project contracts, project pipelines, or a Memorandum of Understanding (MoU) associated with them. A protocol guided the selection of variables for coding and analysis (Table 1). The variables included [Label] (i.e. name of an organisation), [Type] (e.g., a Research Institute; a Private Sector Organisation), [Activity] (e.g., capacity building, development and aid, technology transfer, policy and planning, R\&D and Innovation), [Scale] (e.g., a locally operating organisation, globally operating organisations), [Location] (the city 
Table 1

Research protocol.

Source: produced by authors.

\begin{tabular}{|c|c|c|c|c|c|}
\hline Nodes & Type & Activity & Scale & Location & Description \\
\hline [Organisation] & $\begin{array}{l}{[\text { Intergovernmental Organisation }=[\mathrm{IGO}]]} \\
{[\text { Government }=[\mathrm{GOV}]]} \\
{[\text { Non-Governmental Organisation }=[\mathrm{NGO}]]} \\
{[\text { Private Sector Organisation }=[\mathrm{PSO}]]} \\
{[\text { Knowledge Network }=[\mathrm{KN}]]} \\
{[\text { Local Council }=[\mathrm{LC}]]} \\
{[\text { Research Institute }=[\mathrm{RI}]]}\end{array}$ & $\begin{array}{l}\text { [Capacity Building] } \\
\text { [Development and Aid] } \\
\text { [Education] } \\
\text { [End User] } \\
\text { [Financing] } \\
\text { [Implementation] } \\
\text { [Investing] } \\
\text { [Policy and Planning] } \\
\text { [R\&D and Innovation] } \\
\text { [Technology Transfer] }\end{array}$ & $\begin{array}{l}\text { [Global] } \\
\text { [Regional] } \\
\text { [National] } \\
\text { [Subnational] } \\
\text { [City] } \\
\text { [Local] }\end{array}$ & [Place] & [Operational reach] \\
\hline
\end{tabular}

of the organisation's headquarters and regional offices), and [Description] of relevant context and operational reach.

\subsection{Data analysis}

The analysis included a set of strategies (Table 2). First, we applied qualitative methods of coding, analysis and interpretation of data from the interviews, participant observation and field notes. Secondly, we combined the interview data with secondary data (e.g., government reports, project reports and MoUs), policy analysis (e.g., policy problems, stakeholders, institutional frameworks and uncertainties) and social network analysis (e.g., identifying connections, measuring centrality and selecting a cluster for further analysis).

Of the total network of 198 organisations in our dataset, we ran closeness centrality, betweenness centrality and eigenvector centrality tests using SNA software KUMU and input the data into R Studio to get a random value sample of 100 organisations. We built a network structure and pre-selected the first 20 with highest rank and value for each metric, of which 10 within the highest ranks were clustered, aggregating a total of 15 organisations across the metrics (the most incidental, the direct and indirect nearness between nodes, the information bridge nodes, and the most influential nodes). Based on this selection, we named the 'Hydroclimate cluster' and analysed it to gain an in-depth understanding of their strategies and evaluate their influence in transferring climate and hydrological modelling expertise.

Accordingly, the descriptions of interactions between organisations and events built a global network with which to identify activities and evaluate the performance and behaviour of such connections. Notably, we paid attention to organisations with an active presence in Thailand and Myanmar dealing with climate and hydrological modelling and conducted Social Network Analysis using three measures of network centrality: Closeness centrality, Betweenness centrality and Eigenvector centrality (Scott \& Carrington, 2011). Centrality is a measure of the information about the relative importance of nodes and edges in a graph. Centrality measures the number of links incident on a node and is used to identify nodes that have the highest number of connections in the network. High degree centrality represents a crucial role in the information flow and cohesiveness of the network, thus such nodes are considered central to the network due to their role in the flow of information. We used the three centrality measures mentioned in order to test and increase our confidence of the most relevant organisations under different conditions. We observed marginal variations between the three measures, confirming that: a) the ranks correlate and so do the organisations in direct and indirect nearness, b) the ranks show the leading organisations in information flow, and the ones that act as key knowledge transfer nodes, and c) the ranked organisations are the most prominent given their incidental characteristics. It is important to clarify that we did not consider the results of Social Network Analysis in isolation and that they are not sufficient on their own. Hence, we combined them with our ethnographic data, interviews and policy analysis. The study triangulated the qualitative results based on the interview analysis and conceptual framework with secondary data in order to conduct social network analysis and policy analysis (Table 3 ).

\subsection{Centrality results}

Findings from the network analysis showed a set of organisations with the highest rank values of centrality based on three different analytical metrics. The data showed the elements with the highest degree of connections, being the connectors or hubs. Initial results indicated that the most incidental nodes in the network are as follows: The World Bank, the Climate Technology Centre and Network (CTCN), the Danish Hydraulics Institute (DHI), the Thai Hydro Agro Informatics Institute (HAII), the Thai Science, Technology and Innovation Policy Office (STIPO), Department of Water Irrigation from Myanmar (DWIR), the United Nations Environment Programme (UNEP), the Bangkok Metropolitan Administration (BMA), National Water Rivers Committee of Myanmar (NWRC), the Hydro Informatics Centre from Myanmar, the Global Water Partnership (GWP), and the United Nations Framework Convention on Climate Change (UNFCCC), In addition, we also included data on particular organisations such as the Asia Disaster Preparedness Centre (ADPC), the Green Climate Fund (GCF) and Arcadis, given their relevance in the interviews and secondary data. The interviews, secondary data, and social network analysis indicate that the above organisations are formally connected. Such organisations are linked through formal projects and MoUs and have control over the flow of project data. Based on the research protocol, data analysis and results, we focused our attention on the Hydroclimate cluster in order to gain a thorough understanding of 


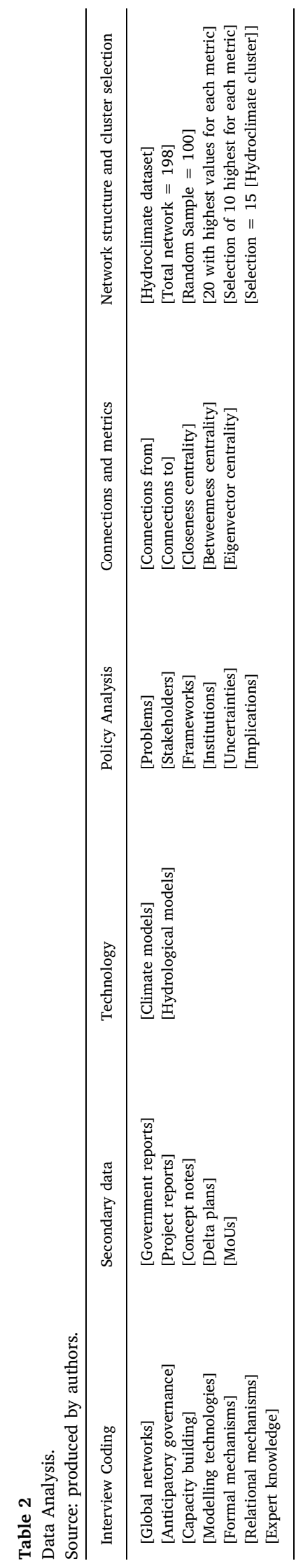


Table 3

Centrality results.

Source: produced by authors.

\begin{tabular}{|c|c|c|c|c|c|c|c|c|}
\hline \multicolumn{2}{|c|}{ Closeness Centrality } & \multirow[b]{2}{*}{ Value } & \multicolumn{3}{|c|}{ Betweenness Centrality } & \multirow[b]{2}{*}{ Rank } & \multicolumn{2}{|c|}{ Eigenvector Centrality } \\
\hline Rank & Label & & Rank & Label & Value & & Label & Value \\
\hline 1 & World Bank & $0.576^{*}$ & 1 & World Bank & $0.247^{*}$ & 1 & World Bank & $0.041 *$ \\
\hline 2 & CTCN & $0.559^{*}$ & 2 & CTCN & $0.243^{*}$ & 2 & DHI & $0.035 *$ \\
\hline 3 & DHI & $0.556^{*}$ & 3 & DHI & $0.195^{*}$ & 3 & CTCN & 0.034 * \\
\hline 4 & MoS\&T_HAII & $0.533^{*}$ & 4 & MoS\&T_HAII & $0.176^{*}$ & 4 & UNEP & $0.031 *$ \\
\hline 5 & STIPO & $0.523^{*}$ & 5 & UNDP & $0.158^{*}$ & 5 & MoTC_NWRC & $0.03^{*}$ \\
\hline 6 & MoTC_DWIR & $0.518^{*}$ & 6 & STIPO & $0.156^{*}$ & 6 & $\mathrm{HIC}$ & $0.03^{*}$ \\
\hline 7 & UNEP & $0.516^{*}$ & 7 & MoTC_DWIR & $0.147^{*}$ & 7 & MoTC_DWIR & $0.028^{*}$ \\
\hline 8 & BMA & $0.496 *$ & 8 & BMA & $0.112^{*}$ & 8 & MoS\&T_HAII & $0.027^{*}$ \\
\hline 9 & MoTC_NWRC & $0.493^{*}$ & 9 & GWP & $0.096^{*}$ & 9 & TM_UNFCCC & $0.026 *$ \\
\hline 10 & HIC & $0.491 *$ & 10 & UNEP & $0.081^{*}$ & 10 & STIPO & $0.024 *$ \\
\hline 11 & UNFCCC & 0.484 & 11 & HIC & 0.072 & 11 & BMA & 0.019 \\
\hline 12 & UNDP & 0.474 & 12 & ICEM & 0.061 & 12 & AIT & 0.018 \\
\hline 13 & GWP & 0.466 & 13 & MoTC_NWRC & 0.061 & 13 & MoTC_DMH & 0.017 \\
\hline 14 & ADPC & 0.462 & 14 & UNFCCC & 0.046 & 14 & UNDP & 0.017 \\
\hline 15 & UNESCAP & 0.462 & 15 & AIT & 0.023 & 15 & ICEM & 0.017 \\
\hline 16 & AIT & 0.461 & 16 & ADPC & 0.018 & 16 & TEC & 0.016 \\
\hline 17 & $\mathrm{ADB}$ & 0.461 & 17 & UNESCAP & 0.018 & 17 & DFAT & 0.015 \\
\hline 18 & MoNREC & 0.451 & 18 & KMS\&T & 0.015 & 18 & GCF & 0.015 \\
\hline 19 & ICEM & 0.446 & 19 & SEIWA & 0.011 & 19 & UNESCAP & 0.015 \\
\hline 20 & Adaptation Fund & 0.435 & 20 & GCF & 0.01 & 20 & Mott Macdonald & 0.014 \\
\hline
\end{tabular}

their strategies and evaluate how they build inferential capacity and governance arrangements for anticipation and environmental planning (Fig. 1).

\section{A global network and the mobilisation of expert knowledge}

\subsection{The hydroclimate cluster}

The Hydroclimate cluster represents an evolving global network with a significant presence in Thailand and Myanmar over the four years observed (2016-2019). It comprises a community of networked organisations linked to the Technology Mechanism of the United Nations Framework Convention on Climate Change (UNFCCC) as well as others participating independently in technology transfer activities (Table 4). The Technology Mechanism was created during the Cancun Agreements in 2010 to accelerate climate technology development and transfer (Olhoff, 2015; UNFCCC, 2011).

Through the creation of National Designated Entities (NDEs), governments such as Thailand obtained accreditation via their National Science, Technology and Innovation Policy Office (STIPO), which serves as the national focal point of the network. STIPO is a government agency that specialises in bridging science, technology and innovation and environmental planning. For example, the organisation developed the National Science, Technology and Innovation Policy Review in 2015 (UNCTAD, 2015), and their National STI Policy and Plan (2012-2021). These efforts include environmental planning objectives on climate change adaptation.

Moreover, STIPO has been playing a strategic role in connecting international organisations such as the Climate Technology Centre and Network (CTCN) and the Danish Hydraulics Institute (DHI), with the Thai Hydro Agro Informatics Institute (HAII) and the Bangkok Metropolitan Administration (BMA), the Green Climate Fund (GCF) and the World Bank ${ }^{1}$. Around the very centre of the Hydroclimate cluster, there is a highly networked set of organisations that serves as a platform enabling the building of relationships with local partners through access to central connections. Most of their services are in the area of capacity building and technology transfer on a project to project basis (CTCN, 2016a). For example, in South East Asia, popular requests include the delivery of technology products in the form of climate and hydrological modelling technologies to government organisations ${ }^{2}$.

One of the most influential nodes in the network is the Danish Hydraulics Institute (DHI). DHI is an international organisation which offers services on climate and water modelling for rivers, deltas, cities and oceans. An essential part of their work is the software development of MIKE models (i.e. MIKE 11, 11 GIS, 21C, Hydro basin, and MIKE 2019), which are globally used by hydrological engineers in public and private organisations (DHI, 2006). DHI has a research department, where they engage and collaborate with universities in different countries, conduct basic research on modelling and develop product portfolios. Recently, they have developed a range of studies on natural hazards in water infrastructure (Oliver, Qin, Larsen, Meadows, \& Fielding, 2018), hydrodynamic data assimilation (Schneider, Ridler, Nygaard, Madsen, \& Bauer-gottwein, 2018) as well as multi-stakeholder scenario tools for decision making using the SIM4NEXUS approach (Sušnik et al., 2018). A crucial network connection is their formal

\footnotetext{
${ }^{1}$ Data from interview with STIPO officials in Bangkok, Thailand, 2017.

${ }^{2}$ Data from interview with CTCN officials in Copenhagen, Denmark, 2017.
} 


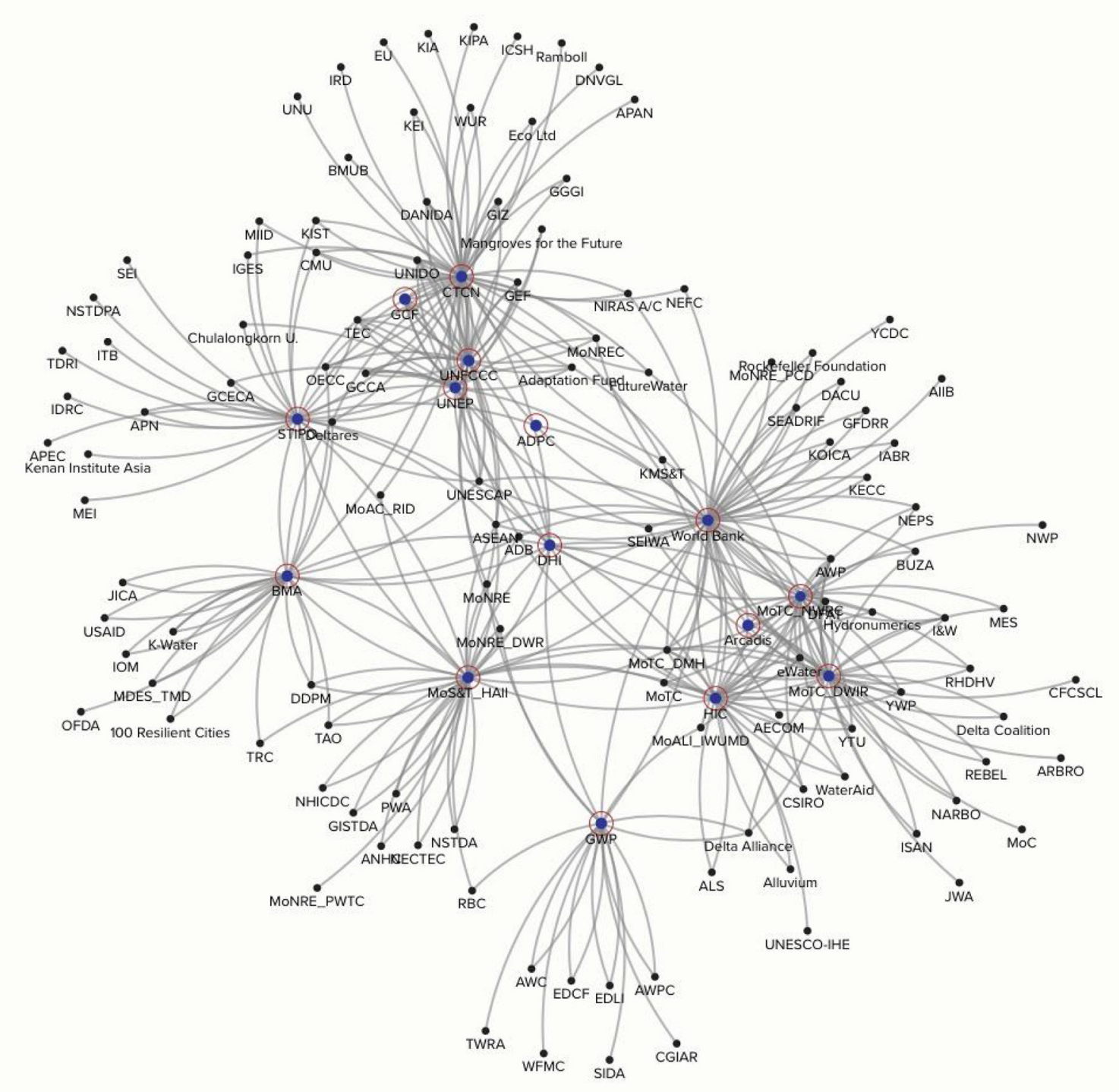

Fig. 1. The Hydroclimate cluster.

Visualisation of global network highlighting in blue with red circle the key organisations with high degree centrality which were selected for analysis.

Source: produced by authors.

partnership with UN Environment, in which the UNEP-DHI Centre on Water and Environment has become an essential part of their water policy and implementation strategy (UNEP-DHI, CTCN, \& UNEP-DTU, 2017). Together with UNEP, DHI has collaborated with the Global Water Partnership (GWP) to advise on the role of Decision Support Systems (DSS) for Integrated Water Resource Management (IWRM). In the resulting report, they provide seven cases of DSS application for water-economic modelling and planning in Asia, South East Asia, Africa and Latin America (GWP, 2013). Since 2017, UNEP-DHI started to follow the UNEP Fresh Water Strategy 2017-2021, which includes a strategic focus on policy and technical advice, capacity development, implementation and strengthening of national legal and institutional arrangements on water management (UNEP, 2017). Consequently, the centre has created a mandate to provide policy advice and technical assistance on water management at the global, transboundary, national, basin and subnational levels. As the implementing agency of UNEP's IWRM Programme, the centre had developed national reports and technology roadmaps for 19 countries to date, of which five are from South East Asia: Cambodia, Laos, Thailand, Vietnam and Myanmar ${ }^{3}$.

In the case of the UNEP-DHI partnership, this constitutes a platform for technology transfer. Specifically, expert knowledge

\footnotetext{
${ }^{3}$ Data from interview with UNEP DHI officials in Copenhagen, Denmark, 2017.
} 


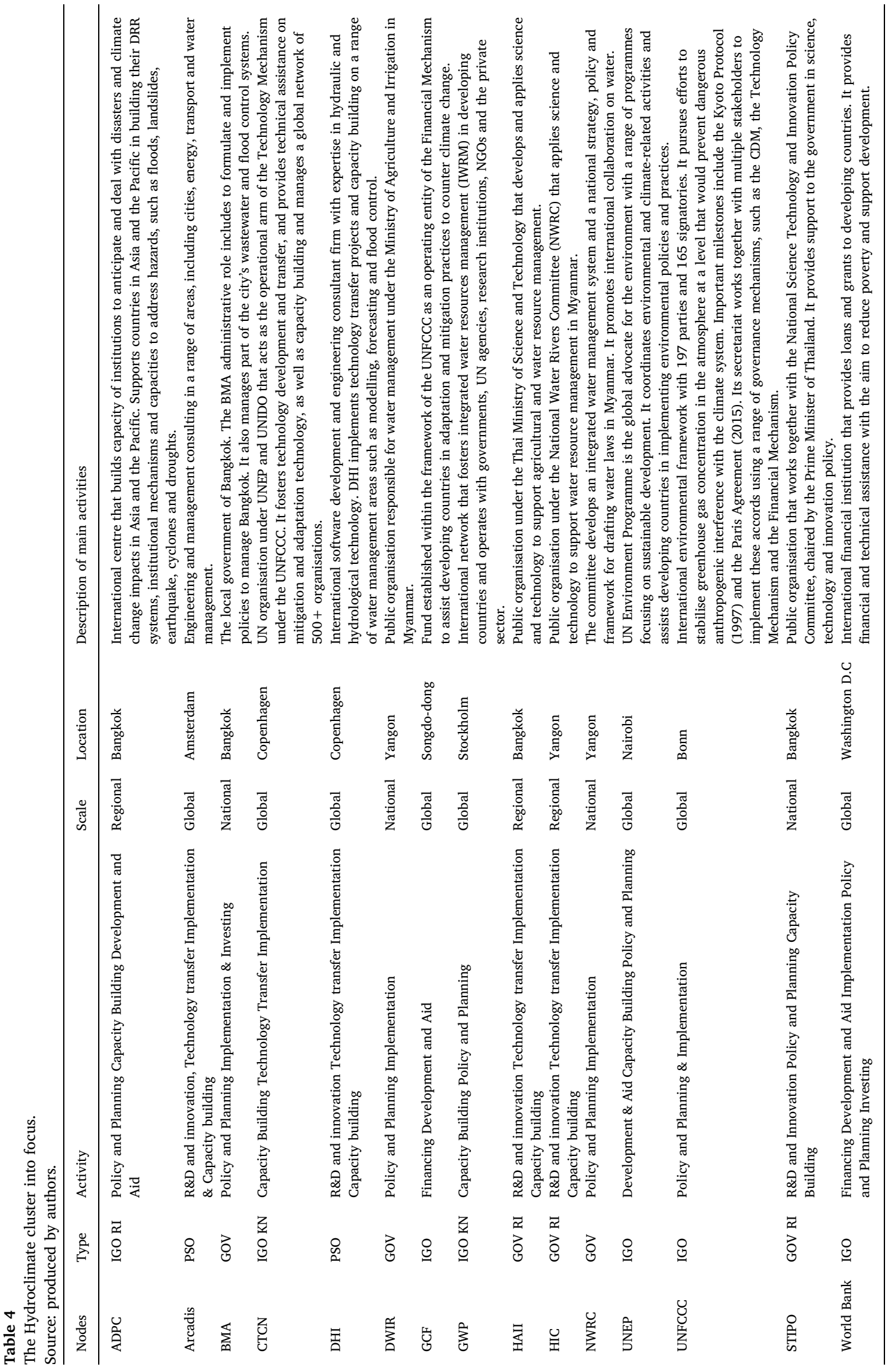


becomes encapsulated in their modelling software. Modelling software such as MIKE enables water engineers to do forecast as well as scenario analysis for project-specific future conditions to support decision makers ${ }^{4}$. Further, the organisation has developed specific guidelines for climate and water systems interactions in urban and rural contexts and coastal areas. The guidelines combine DHI's modelling approach with recommendations of how to deal with water resource management challenges in projects, considering future climate projections and operational challenges for decision making ${ }^{5}$. For example, UNEP-DHI has been working for the past ten years in Thailand with different organisations and was involved when the flooding in the Chao Phraya occurred in 2011. Their response on that occasion was to establish a collaboration with the government to build a climate and water forecasting system for the Bangkok Metropolitan Administration (BMA). As a strategy, they delivered an operational MIKE system for Bangkok. Through a capacity building scheme, they deployed the software and trained local staff (CTCN, 2015). In Thailand, the Hydro Agro Informatics Institute (HAII) have been active for some time and have been recipients of DHI modelling technologies and operational training. As part of the response plan after the 2011 floods, HAII has been collaborating with BMA in the building of water statistics. In the case of Myanmar, the strategy of the national government has been to engage with the World Bank and Asian Development Bank (ADB) and share lessons with the HAII in Thailand. DHI, for example, has supported the development of the Thai HAII and them realising their ambition to become a regional expert centre on water statistics and water management services. As a result, there is a collaboration between DHI and HAII with a focus on supporting the government of Myanmar ${ }^{6}$. In the next subsection, we examine the operational strategies and organisational linkages with a focus on the mobilisation of capacity building through project-based interactions.

\subsection{Operational strategies and project-based interactions}

The establishment of strategic alliances in the Hydroclimate cluster, for example between UN Environment (UNEP) and the Danish Hydraulics Institute (DHI), has been operationalised through the transfer of climate and hydrological modelling technologies on a project to project basis. The global network, managed by the CTCN, matches demand and supply sides and provides technical guidance on technologies and funding ${ }^{7}$. The strategic position of the UNEP-DHI partnership allows proximity on interactions with CTCN and other UNFCCC related agencies, including those of the Financial Mechanism (e.g., GCF). These strategic linkages enable the means for implementation through tailored portfolios of expertise. These portfolios contain project ideas and concept notes arising from network clients (e.g., BMA). The process involves the pairing of potential solutions to requests with funding and prioritised sectors and technologies (e.g., through Technology Needs Assessments). For example, the Bangkok Metropolitan Administration has been in the CTCN country list and is a network member. The BMA needed to obtain hydrological modelling software and to build local capacity to operate such technology.

Consequently, the BMA selected DHI to be the implementer of an Early Warning System for the city of Bangkok (CTCN, 2015). While other organisations such as Arcadis and Deltares bid for the same projects, there was a previous history of project-based interactions between DHI and BMA. Hence, the level of confidence that DHI would effectively supply the service was higher, and the project feasibility studies went immediately to technical details of implementation ${ }^{8}$. In this case, the interactions between DHI and BMA are based on a combination of formal governance as well as relational mechanisms that evolve through project-based interactions. As a result, established links in highly networked organisations maintain institutional dynamics that are legitimised through formal and relational strategies.

As formalities developed into concrete Memorandums of Understanding (MoUs), the funding applications ran in parallel. Furthermore, DHI and BMA worked together in developing a concept note and implementation plan which the CTCN agreed to support. Operations continued with DHI engaging with STIPO, the National Designated Entity (NDE) of the Technology Mechanism in Bangkok. The NDE for Thailand has high credibility from the Thai government for being both a scientific and a policy organisation which advice the cabinet office ${ }^{9}$. DHI decided to get involved with the NDE from the initial project description phase to the drafting process a year in advance of the bidding process for funding ${ }^{10}$. The practice was coherent for the stakeholders involved (i.e. UNEP, DHI, BMA, STIPO) and the regional CTCN officers at the United Nations facilities in Bangkok. This example illustrates how an organisation uses access to the network to maintain its operational reach. The strategic positioning offers network members a direct line for project implementation at city levels such as in Bangkok, Jakarta and Yangon. Project-based interactions can take the form and length of short training sessions of two weeks up to technology transfer implementation trajectories of two years. This strategy combines a broader portfolio of technology options and includes policy and implementation phases ${ }^{11}$. The network interactions described represent long term engagement through project-based interactions that link technical engineering aspects of tools (e.g., in the case of modelling technologies), with policy advice and capacity building. This combination of formal arrangements with relational mechanisms makes the UNEP-DHI partnership one of the most active hydrological modelling companies in Thailand.

Myanmar, on the other hand, is a new evolving market of competitors. In the observed period, the World Bank dominated most

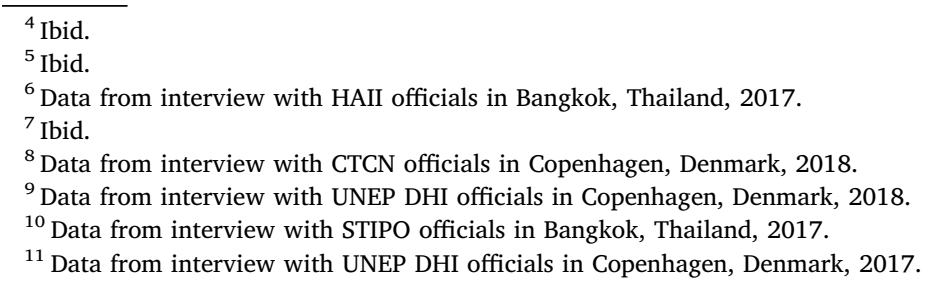


hydrological projects in Myanmar and organisations such as Arcadis and Deltares were actively trying to position themselves against their competitor DHI. However, as Myanmar is still uncharted territory, DHI used their organisational links and established a local office in Yangon. Over the last two years (2016-2018), their operations have had a focus on building relations with the national government and developing a project portfolio together, while Myanmar is requesting technical assistance from the CTCN to build data for climate change, drought and flood management for the country with DHI as the technology implementor (CTCN, 2016b). From the financial point of view, the Green Climate Fund (GCF) requires countries to reach a readiness status that comes as a combination of capacities that together enable the development of bankable concept notes for technology transfer funding (GCF, 2018). For example, one of most demanded projects by the government of Myanmar has been the development of capacity for climate change adaptation through systems that combine real-time climate data with hydrological data in a type of information portal which can guide decisionmakers ${ }^{12}$. The government is pursuing efforts to combine strategies for resilient agricultural development using such tools. As this section has shown, networked organisations take key operational strategies such as tailored technology portfolios built from continuous project-based interactions. The mobilisation of technological packages in the form of modelling technology comes with designed capacity building schemes that can be replicated and scaled up from country to country ${ }^{13}$.

\section{Anticipatory governance and authoritative knowledge in practice}

As seasonal variability increases in deltas, demonstrated in the 2011 floods in Thailand, anticipating events requires enough preparation in terms of paying attention to cumulative events and systemic feedbacks from the environment as well as effectively managing institutions. For example, in 2011 the Asian Disaster Preparedness Centre (ADPC) anticipated an increase in storm frequency and changes in rain patterns. Warnings of not enough preparation for disaster scenarios were not considered by the government, particularly on draining water from the upstream of the delta, which was suggested by the ADPC. The reservoirs were at their maximum capacity before the rainy seasons, and when storms came, the infrastructure could not hold more water. Furthermore, the institutional management of the problem was characterised by poor coordination amongst government departments, while some competing agencies were trying to do the same activities with no guidelines or instructions and the Department for Disaster and Mitigation had no decision-making role, but merely that of advisor ${ }^{14}$.

The Asian Disaster Preparedness Centre (ADPC) as a regional disaster management agency, has a role in producing tools based on GIS, remote sensing and satellite technology to support governments in South East Asia at the national and subnational level. Their role is twofold. They provide scientific evidence and policy advice to decision makers, for example, in Thailand and Myanmar. Their activities include analysing historical data, building models and trying to predict what would be the likelihood of particular events happening, considering potential impacts on infrastructure and population. Financial support for this organisation comes from the United States Agency for International Development (USAID), and it receives technical assistance from the National Aeronautics and Space Administration (NASA). As a networked organisation, ADPC is in charge to build evidence to inform decision making in the region, by analysing multiple forms of data and translating results to non-technical audiences. Their expertise focuses on disaster risk management and uses DHI's MIKE for hydrology, combined with ground motion modelling (for earthquakes) and other tools such as General Circulation Models (GCMs). Together they liaise with government organisation such as the Thai Hydro Agro Informatics Institute (HAII) and recently with the Hydro Informatics Centre (HIC) in Myanmar ${ }^{15}$.

\section{1. (In)action based on evidence?}

There are many challenges associated with relying on climate change scenarios and downscaling them at project levels in a specific context, especially regarding climate change impacts. In recent years, there have been efforts in R\&D to purposely design downscaling of global climate data and try to match this with local climate and water data. One of the main challenges is the reliance on GCMs and then interpreting such data in different contexts. The statistical downscaling needs different methodologies. For example, DHI and ADPC use the input of new climate conditions into MIKE models to study their interactions. The starting point is to look at the chain of different data inputs from global, regional and local scenarios. Then they combine impact assessments and adaptation analysis. In each step, there will be different uncertainties that need to be considered, which is tested through simulations and models that can translate data into statistical terms by determining probability distributions. The results are then used as a 'knowledge chain' for bias correction when applying it to a hydrological model ${ }^{16}$. What expert organisations such as DHI and ADPC recognise is that the knowledge chain quickly becomes a chain of uncertainties. For example, in Bangkok, the implementation of MIKE needed to demonstrate what the software can and cannot do by showing to the BMA how the model works. The capacity building requires to explain its potential as well as limitations, leading to reflective workshops with local experts, and to overcome some of the anticipatory myths surrounding modelling technologies. Reflecting on limitations enables the inferential capacity of the local organisation to develop by deploying the technology together with the institutional guidelines for its operations. One of the DHI experts in Bangkok commented on such challenges:

\footnotetext{
${ }^{12}$ Data from interview with UNEP DHI officials in Copenhagen, Denmark, 2017.

${ }^{13}$ Data from interview with DHI officials in Bangkok, Thailand, 2017.

${ }^{14}$ Data from interview with ADPC officials in Bangkok, Thailand, 2017.

${ }^{15}$ Data from interview with HAII officials in Bangkok, Thailand, 2017.

${ }^{16}$ Data from interview with DHI officials in Copenhagen, Denmark, 2018.
} 
"Some of the main challenges of implementing such [modelling] tools are the overall accuracy of the model. If it is not accurate, predictions will not be accurate either. Then there is the driving input, such as rainfall data, which is a challenge. The information we get here [Bangkok] is delayed in time. If you are trying to anticipate what could happen in the next $6 \mathrm{~h}$ and the data you used to make such prediction has latency, by the time it reaches your computer it is already $5 \mathrm{~h}$ old. Then the whole forecast is unreliable, if at all relevant to a particular decision. It is key to explain these potential dynamics when you are training local engineers. "17

Moreover, the technical challenges are doubled up by institutional dimensions that are fundamental in performing anticipatory governance. One of the ADPC experts commented:

"The problem is that there is no integration of different agencies dealing with water management in South East Asia. It is the same in Thailand, Vietnam and Myanmar. Because water is such a key asset, everyone wants to be involved and have a say. There are so many overlapping mandates in Thailand for example, regarding water management. Same in Myanmar. A lot of players claiming that they are capable of doing this big job alone, which is not the case. You need to collaborate with other agencies, not compete. Collaboration, data access and interpretation are one thing, then acting based on evidence is another."18

This context shows the complexity of building the capacity to manage climate and hydrological data, building evidence and using such knowledge to inform decision making in South East Asian countries. When it comes to governing the processing of raw data and transferring it from one platform or institutional context to another (from the scientific to the policy context), it becomes critical how the knowledge boundaries are maintained through performative expertise. Therefore, there is a need for concrete frameworks to guide policymakers, which are required to be technically articulated and understandable at the same time. This something that expert agencies typically fail to do. Besides, in Thailand and Myanmar, very few want to claim authoritative knowledge to make final decisions when it comes to disaster prevention and management. However, when protocols are established ex ante, a governance arrangement follows involving anticipation and scenario planning, as clear instructions protect individuals and allow them to perform sound decisions. The relational component, as ADPC has shown, has to do not just with building the technical expertise, but involves convincing the decision-making level to trust a guided response that has been anticipated and coordinated at the institutional level. On the technical level, the capacity to enable standardised mechanisms that speed up data flow between organisations was one of the recommendations taken up by the BMA when DHI implemented the Early Warning System for Bangkok. The issue of data accessibility would need to be overcome by further coordination between the BMA and the HAII. However, the evident institutional fragmentation makes the governance of climate and hydrological data difficult. It needs to be translated from one system to another before it is ready to use. As a result, chains of data stations have to be aligned to the correct data flow. As a "learning for future projects", DHI concluded that BMA could recalibrate and use weather radars to improve data flow and accuracy and upgrade their infrastructure. They also suggested to continuously run simulations and use scenario tools to anticipate what could happen if particular patterns emerge and learn how to proceed if conditions are not met in the system.

\subsection{More than tools and techniques}

Using a strategic engagement process through continuous capacity building, networked organisations provided local organisations with the possibilities, challenges and roadmaps for future project interactions. However, focusing only on technical expertise does no more than adding the use of tools to management procedures. Governing anticipatory practices requires coherent governance capacities in order to enable coordination and data sharing between the stakeholders. It will continue to be challenging for government agencies to use the tools and techniques effectively if not combined with sound institutional coordination. The results show that local governance dynamics make expertise harder to reach authority, ultimately rendering inferential capacities not sufficient to influence decision-making processes aimed at managing risk. Having tools to forecast and techniques to represent the likelihood of occurrence of hazardous events is only beneficial if the relevant organisations also have clear operational response guidelines to steer preventive actions. One of the weakest points in the development of inferential capacities indeed is the ability to access, downscale and integrate reliable climatic and hydrological data in real time in order to anticipate near future events, especially when such data belongs to various sources with fragmented mandates that overlap or compete.

Our analysis shows how the Hydroclimate cluster composed of globally networked organisations develop project-based interactions to deploy tools and techniques. The data also suggest that particular forms of expertise - the development of inferential capacities to anticipate and plan disaster management - is a salient activity in Thailand and Myanmar. However, capacity building for anticipatory governance in climate change adaptation is twofold. On the one hand, it requires the development of the technical capacities to operate tools such as climate and hydrological models. On the other hand, in order to have governance effects, inferential capacities need a degree of legitimacy, enough to produce institutional action. By 'institutional action', we mean concrete responses in the short term via specific protocols and guiding principles to national organisations, and in long-term environmental planning. Furthermore, anticipatory practices need a combination of the modelling tools that are dependent on climate, weather and water data as combined inputs in adaptation and risk analysis. In principle, the input allows national agencies in South East Asia to infer short-term interactions of socio-ecological systems in the deltas (e.g., to monitor flood risk). However, their success depends on the balancing of tools and expertise, of preventive actions and operational procedures to act when is required.

\footnotetext{
${ }^{17}$ Quote from interview with DHI officials in Bangkok, Thailand, 2017.

${ }^{18}$ Quote from interview with ADPC officials in Bangkok, Thailand, 2017.
} 
Project pipelines from networked organisations in water and agriculture in South East Asian deltas are only increasing. So far, identifying organisations belonging to a formally arranged global network has enabled us to track and analyse examples of how capacity building for anticipatory governance operates in practice and illustrate the complex interactions between actors, technology and institutions. We also illustrated many limitations and lessons regarding the capacity to manage knowledge boundaries in order to build a legitimate transfer of operational frameworks for risk management.

\section{Discussion and conclusion}

Capacity building to make inferences about future development is relevant in environmental planning. The adaptive capacity of government organisations varies from context to context, in their technical ability to use modelling tools, as well as in their institutional capacity to generate authoritative responses. In this article, we examined how networked organisations attempt to build capacity for anticipatory governance through project-based interactions in Thailand and Myanmar. Findings suggest that although particular organisations manage to centralise the mobilisation, transfer and operational capacity of modelling technologies due to formal and relational mechanisms, their governance effect at the local level is limited. As the study shows, the mobilisation of anticipatory governance tools continues to spread across the Hydroclimate cluster in South East Asia, and yet the institutional challenges at the local level, the political conditions and the ability of local organisations to elaborate robust inference with which to inform decision making continue to be challenging factors. The persistence of particular forms of networked organisations explains the presence of de facto governance practices. It reveals the generation of legitimacy in relational mechanisms through project-based interactions and shows how a cluster of organisations build a collaborative platform. However, the consolidation of such practices does not yet guarantee its effectiveness with respect to dealing with climate change adaptation. It further demonstrates that technology transfer projects need to be backed up with social and strategic capacity building in order to nurture consistent anticipatory governance in different cultural contexts.

Our study shows that expert knowledge and skills are not easily transferrable in the context of climate and water governance. Knowledge sharing, capacity building and concrete preventive actions are at least equally important as technology transfer projects in climate change adaptation and risk management. However, as this article demonstrates, balancing technology and capacity building is not yet a standard practice. Cases from Thailand and Myanmar illustrate some of the real challenges when dealing with anticipatory governance tools and techniques in different cultural settings. For those reasons, we argue that consolidated networks that de facto legitimise expertise in climate and water governance, fall short in effectively building capacity to govern future climate change risks. The mismatch happens in part because of short-term, one-off technology transfer projects that are unbalanced in practice, especially concerning their complex institutional dimensions. Consequently, current technology transfer programmes in South East Asia do not necessarily translate into an enhanced institutional capacity for environmental planning. Further, as the article shows, project-based technological fixes continue to be high on the agenda of development cooperation networks as the way to solve adaptation challenges. However, continuing to favour such practices over institutional capacity building undervalues the complex social dimensions of technology, and thus hinders the adaptability of organisations dealing with climate change and water governance. Adaptation governance should therefore pay more attention to the institutional dimension. In this context, the mobilisation of expert tools and techniques needs to re-consider the inherent differences between the sites of knowledge production and the sites of knowledge use.

On the practical side, global networks dealing with anticipatory governance practices could better use their relational properties and authoritative expertise to build capacity in two areas. First, by focusing on preventive actions. By this, we mean building ex-ante institutional practices aimed to decrease risk by conducting regular scenario exercises and use modelling tools to plan and build resilient mechanisms to prevent floods. And second, by supporting the development of transparent operational response measures. This entails the development of concrete arrangements and building of institutions prepared to manage potential disasters. These measures include planning emergency budgets and the governance of institutional capacity to deal with operations, following clear and transparent mandates that coordinate the relevant national agencies. Organisations in Thailand and Myanmar could further align efforts in developing standardised responses linked to national adaptation plans. This adaptation capacity could enable, with the support of global strategic alliances, an enhanced configuration of techniques and tools.

As this research has demonstrated, anticipatory governance is a combination of foresight knowledge and authority, by which organisations can assist and encourage policy-makers to envision the potential consequences of their actions while providing frameworks to evaluate risk and opportunity. Anticipatory governance practices need the balancing of foresight tools and techniques with sound institutional arrangements in order to be more effective. Policy-makers need to be prepared for potential scenarios, collaborate with expert modelling organisations and act preventively on climate change adaptation and risk management. We conclude that preventive actions, together with transparent operational response frameworks, could significantly improve resilience and adaptability of local knowledge systems and institutions dealing with climate change adaptation. Such integration could enable anticipatory response measures to better manage risk, as well as increase institutional cooperation for long term environmental planning.

\section{Declaration of Competing Interest}

The authors declare no conflict of interest. 


\section{Acknowledgements}

The authors would like to thank the participants in the project and the research team. We thank Dr Ine Steenmans for her valuable feedback to our manuscript. We also thank the anonymous reviewers for their time and essential feedback. The project was funded by the Economic and Social Research Council (ESRC) [grant number ES/N018834/1] of the United Kingdom, in collaboration with the Netherlands Organisation for Scientific Research (NWO) [grant number 464-15- 086] and was part of a joint collaboration between Osaka University, University of Amsterdam, IRD, CIRAD, UNESCO-IHE and UCL STEaPP under the Open Research Area (ORA) for the Social Sciences Agreement.

\section{References}

Aerts, J., Botzen, W., Bowman, M. J., Ward, P. J., \& Dircke, P. (2012). Climate change adaptation and flood risk in coastal cities. Earthscan. New York: Routledge. Arkadani, M. R. M., Hashemi, S. M., \& Razazzi, M. (2019). A Cloud-based solution/reference architecture for establishing collaborative networked organisations. Journal of Intelligent Manufacturing, 30, 2273-2289.

Betsill, M. M., Bulkeley, H., \& Mcguirk, P. (2004). Transnational networks and global environmental governance: The cities for climate protection program. International Studies Quarterly, 48(2), 471-493.

Boston, J. (2017). Governing for the future. Bingley, England: Emerald.

Camarinha-Matos, L. M., \& Afsarmanesh, H. (2005). Collaborative networks: A new scientific discipline. Journal of Intelligent Manufacturing, 16, 529-542.

Clark, W. C., Tomich, T. P., van Noordwijk, M., Guston, D., Catacutan, D., Dickson, N. M., \& McNie, E. (2016). Boundary work for sustainable development: Natural resource management at the Consultative Group on International Agricultural Research (CGIAR). Proceedings of the National Academy of Sciences of the United States of America, 113(17), 4615.

CTCN. (2015). Response_plan_-_bma_urban_flood_early_warning_demonstration_sysytem_201500085_signed_by_all.pdf. Report, Copenhagen, Denmark.

CTCN (2016a). Global climate commitments 2016 in actionCopenhagen, Denmark: Report.

CTCN (2016b). Response_plan_myanmar_signed.pdfCopenhagen, Denmark: Report.

DHI (2006). MIKE 21 \& MIKE 3 flow model FM hydrodynamic modelCopenhagen: Report.

Enserink, B., Kwakkel, J. H., \& Veenman, S. (2013). Coping with uncertainty in climate policy making: (Mis)understanding scenario studies. Futures, 53, 1-12.

Fischer, M. (2015). Anthropology of science and technology. Boston: MIT Cambridge.

Fuerth, L., \& Faber, E. (2012). Anticipatory governance: Practical upgrades - Equipping the executive branch to cope with increasing speed and complexity of major challenges.

Washington, DC: Elliot School of international Affairs: George Washington University.

Funtowicz, S., \& Ravetz, J. R. (1994). Emergent complex systems. Futures, 26(6), 568-582.

GCF (2018). GCF handbook: Decisions, policies and frameworksReport.

Gupta, A., Biermann, F., Andresen, S., \& Bernd, S. (2012). Science networks. In Pattberg (Ed.). Global environmental governance. Cambridge Massachusetts: The MIT Press.

Gupta, A., \& Möller, I. (2019). De facto governance: How authoritative assessments construct climate engineering as an object of governance. Environmental Politics, 28(3), 480-501.

Guston, D. H. (2014). Understanding 'anticipatory governance.'. Social Studies of Science, 44(2), 218-242.

GWP (2013). The role of decision support systems and models in integrated river basin managementReport.

Haas, P. M. (1992). Introduction: Epistemic communities and international policy coordination. International Organization, 46(1), 1-35.

Hajer, M. A., \& Pelzer, P. (2018). 2050—An Energetic Odyssey: Understanding 'Techniques of futuring' in the transition towards renewable energy. Energy Research \& Social Science, 44(1), 222-231.

Hogendoorn, D., Zegwaard, A., \& Petersen, A. C. (2018). Difficult travels: Delta plans don't land in the Chao Phraya delta. Environmental Science and Policy, 89, 378-384. IPCC (2014). AR5 climate change 2014: Synthesis report. In Core Writing Team, R. K. Pachauri, \& L. A. Meyer (Eds.). Contribution of working groups I, II and III to the Fifth assessment report of the intergovernmental panel on climate change. Geneva, Switzerland: IPCC.

IPCC (2018). IPCC Special Report (15) on $1.5^{\circ} \mathrm{CG}$ eneva, Switzerland: IPCC.

Kypreos, S., \& Turton, H. (2011). Climate change scenarios and technology transfer protocols. Energy Policy, 39(2), 844-853.

Mahoney, M., \& Hulme, M. (2012). Model migrations: Mobility and boundary crossing in regional climate prediction. Transactions of the Institute of British Geographers, 37, $197-211$.

Marcus, G. E. (1995). Ethnography in/of the world system : The emergence of multi-sited ethnography. Annual Review of Anthropology, $24(1), 95-117$.

Morita, A. (2014). The ethnographic machine: Experimenting with context and comparison in strathernian ethnography. Science, Technology \& Human Values, 39(2), 214-235. Olhoff, A. (2015). Adaptation in the context of technology development and transfer. Climate Policy, 15(1), 163-169.

Oliver, J., Qin, X. S., Larsen, O., Meadows, M., \& Fielding, M. (2018). Morphological dynamics and dike failure. Natural Hazards, 91(1), $287-307$.

Ottaway, M. (2001). Corporatism Goes global: International organizations, nongovernmental organization networks, and transnational business. Global Health Governance: the Scholarly Journal for the New Health Security Paradigm, 7(3), 265-292.

Rip, A. (2010). De facto governance of nanotechnologies. In M. Goodwin, B.-J. Koops, \& R. Leenes (Eds.). Dimensions of technology regulation (pp. 285-308). The Netherlands: Wolf Legal Publishers.

Sardar, Z. (2010). The Namesake: Futures; futures studies; futurology; futuristic; foresight-What's in a name? Futures, 42(3), 177-184.

Scheel, C. (2002). Knowledge clusters of technological innovation systems. Journal of Knowledge Management, 6(4), 356-367.

Schneider, R., Ridler, M., Nygaard, P., Madsen, H., \& Bauer-gottwein, P. (2018). A data assimilation system combining CryoSat-2 data and hydrodynamic river models. Journal of Hydrology, 557(1), 197-210.

Scott, J., \& Carrington, P. (2011). The SAGE handbook of social network analysis. London: SAGE.

Stone, D., \& Maxwell, S. (2005). In D. Stone, \& S. Maxwell (Eds.). Global knowledge networks and International development. New York: Routledge.

Stone, D. (2003). Knowledge networks and global policy. global networks. Conference Paper, CEEISA/ISA Conference, Vol. 2.

Stone, D. (2008). Global public policy, transational policy communities and their networks. Policy Studies Journal, 36(1), 19-38.

Sušnik, J., Chew, C., Id, X. D., Mereu, S., Trabucco, A., Evans, B., ... Savi, D. A. (2018). Multi-stakeholder development of a serious game to explore the water-energy-Food-LandClimate Nexus : The SIM4NEXUS approach. Water, 10(139), 1-21.

Tsing, A. L. (2005). Friction: An ethnography of global connection. Princeton University Press.

UNCTAD (2015). Science, technology and innovation policy review: Thailand. Geneva, Switzerland: United Nations.

UNEP-DHI, CTCN, \& UNEP-DTU (2017). Climate change adaptation technologies for waterJoint Report, Copenhagen, Denmark.

UNEP (2017). Freshwater strategy 2017-2021 https://wedocs.unep.org/bitstream/handle/20.500.11822/19528/UNEP-full_report-170502.pdf? sequence = 3\&isAllowed =y.

UNFCCC (2011). Report of the Conference of the Parties on Its Sixteenth Session, Held in Cancun from 29 November to 10 December 2010. Decision 1/CP.16.

van Asselt, H. (2013). The fragmentation of gloabl climate governance: Consequences and management of regime interactions. Amsterdam: Wormann Print.

van Kerkhoff, L. (2013). Knowledge Governance for Sustainable Development: A Review. Challenges in Sustainability, 1(2), 82-93.

Vermeulen, S. J., Challinor, A. J., Thornton, P. K., Campbell, B. M., Eriyagama, N., Vervoort, J. M., \& Smith, D. R. (2013). Addressing uncertainty in adaptation planning for agriculture. Proceedings of the National Academy of Sciences, 110(21), 8357-8362.

Vervoort, J., \& Gupta, A. (2018). Anticipating climate futures in a $1.5{ }^{\circ} \mathrm{C}$ Era: The link between foresight and governance. Current Opinion in Environmental Sustainability, 31 , $104-111$.

Wadell, S. (2011). Global action networks: Creating our future together. Milan: Bocconi University Press.

Young, O. (2017). Governing complex systems. Social capital for the anthropocene. USA: MIT Press.

World Bank (2012). Thai flood 2011: Rapid asessment for resilient recovery and reconstruction planning. Bangkok Office: The World Bank. 